\title{
NANOMAGNETISM AND DOMAIN STRUCTURE OF SINTERED NICKEL-ZINC FERRITES STUDIED BY MAGNETIC FORCE MICROSCOPY
}

\author{
Anderson Dias \\ Departamento de Engenharia Metalúrgica e de Materiais \\ Universidade Federal de Minas Gerais, Rua Espírito Santo 35, Sala 206, Belo Horizonte-MG, \\ 30160-030, BRAZIL \\ E-mail: anderson@demet.ufmg.br
}

The nanomagnetic structure of hydrothermal $\mathrm{NiZn}$ ferrites sintered at various temperatures was studied in this work through Magnetic Force Microscopy using different magnetically characterized tips. The magnetic patterns varied according to the tip employed and were correlated to the magnetic induced field generated by these different coated tips. Nickel ferrites were produced at $200^{\circ} \mathrm{C}$ by hydrothermal synthesis and sintered at temperatures ranging from $1100^{\circ} \mathrm{C}$ to $1400^{\circ} \mathrm{C}$. Ceramic bodies were obtained with final grain sizes between 3 and $30 \mathrm{~m}$, porosities of $8-3 \%$, and composition $\mathrm{Ni}_{0.34} \mathrm{Zn}_{0.60} \mathrm{Fe}_{2.06} \mathrm{O}_{4}$ [1]. A commercial MFM from Digital Instruments, Inc., operating in Tapping and Lift Modes was employed to study the topographic and magnetic features of the samples. The topographic contribution to the magnetic image was eliminated by using two interleaved scans, and both the topographic and the magnetic image could be storage simultaneously. The signal from the mechanically modulated cantilever was analyzed in a lock-in amplifier and the phase shift, proportional to the force gradient, is recorded. NanoProbe ${ }^{\mathrm{TM}}$ magnetic coated tips were employed in frequency mode for the magnetic characterization: (i) low-coercivity-LC (Fe-based coating); (ii) lowmoment-LM (15 nm-thick Co alloy coated); and (iii) high-coercivity-HC (Co alloy coated) tips with resonant frequencies in the range $60-100 \mathrm{kHz}$, spring constant of $1-5 \mathrm{~N} / \mathrm{m}$, and cantilever length of $225 \mathrm{~m}$.

Figure 1 displays AFM images of the NiZn ferrites hydrothermally synthesized at $200^{\circ} \mathrm{C}$. Nanometer sized particles (below $50 \mathrm{~nm}$ ) can be seen in both topographic (left) and phase (right) images. Figure 2 presents topographic and magnetic images of a NiZn ferrite sample sintered at $1100^{\circ} \mathrm{C}$, for 4 hours. At this temperature, the grain size remains constant $(3 \mathrm{~m})$ for all times studied (5-240 minutes) and high porous ceramics were obtained [1]. MFM characterization shows a nanosized domain structure, as expected. Samples sintered at temperatures above $1200^{\circ} \mathrm{C}$ present an remarkable grain growth $(10-30 \mathrm{~m})$. The transition from open porosity to intergranular and, finally, intragrains was observed in samples sintered at 1200,1300 and $1400^{\circ} \mathrm{C}$, respectively. The results indicate the higher influence of the densification rate than that of grain growth rate in the sintering of NiZn ferrites [1,2]. Figure 3 presents topographic and magnetic images for samples sintered at 1300 and $1400^{\circ} \mathrm{C}$. As it can be seen, different magnetic patterns are observed as a result of the fine domain structure in these materials even for grains in the order of $10 \mathrm{~m}$ or more.

Figure 4 displays a sequence of images (topographic plus magnetic) obtained at a tip-sample distance equal to $200 \mathrm{~nm}$ and under different magnetic induced fields: (i) LC probes (coercivity < 10 Oe - Fig.4a), (ii) LM probes (coercivity < 400 Oe - Fig.4b), and (iii) HC probes (coercivity $>4,000 \mathrm{Oe}-\mathrm{Fig} .4 \mathrm{c}$ ). The goal was to verify if changing the strength of the induced magnetic signals was possible to influence the magnetic structure of the ferrites. The results showed that different magnetic contrast, with dark and bright regions representing domains of opposite magnetization can be visualized depending upon the magnetic tip employed. In our imaging process, the tip, magnetized perpendicular to the surface of the sample, interacted with the gradient of the $z$-component of the sample's stray field within a dipolar approximation of the tip, which caused a shift in the resonant frequency of the 
cantilever. As expected, the tip interacted with the oppositely magnetized domains by being either attracted or repelled from the sample surface.

For LC tips (Fig. 4a), the magnetic structure was not well defined and only the contrast of the domain walls can be observed. First, it can be explained by the fact that the maximum value for the force attracting a tip to the surface occurs above the domain wall, where the gradient of the stray field is the largest (if you consider the anisotropy energy of the tip and the interaction energy between the tip and the sample). Also, the tip presents a relatively high moment $\left(10^{-13} \mathrm{emu}\right)$ that perturbs the magnetic domains of the sample in the vicinity of the domain walls. On the other hand, LM tips (Fig. 4b) provided sharper images with better lateral resolution if compared with the patterns observed when LC tips were used. Thus, we can assume that tips with low magnetic moment and relatively high coercivity are most adequate to detect the magnetic domains of the ferrites studied in this work. It was also observed that there is a strong correlation between the domain walls imaged with the MFM and the dissipation signal. Fig. $4 \mathrm{c}$ shows the magnetic structure and domains of the NiZn ferrites obtained by using HC probes. In this case, we have a combined effect of both highcoercivity $(4,000 \mathrm{Oe})$ and relatively high moment $\left(10^{-13} \mathrm{emu}\right)$ acting on the magnetic structure of our samples. The final image presents well-defined domains but with strong "perturbations": the domain walls near to the scratch cannot be clearly seen and only "strips" are visible; the lateral resolution is thus very poor. It is important to note that the well known enhance in the resolution by decreasing the tip/sample distance was destroyed by the high magnetic stray field of the probe at the surface of our magnetically soft sample, and the magnetic structure was seriously perturbed.

\section{References:}

[1] A. Dias; R.L. Moreira; N.D.S. Mohallem. J. Phys. Chem. Solids 58 (1997) 543.

[2] A. Dias; R.L. Moreira; N.D.S. Mohallem; A.I.C. Persiano. J. Magn. Magn. Mater. 172 (1997) L9.

\section{Figures:}

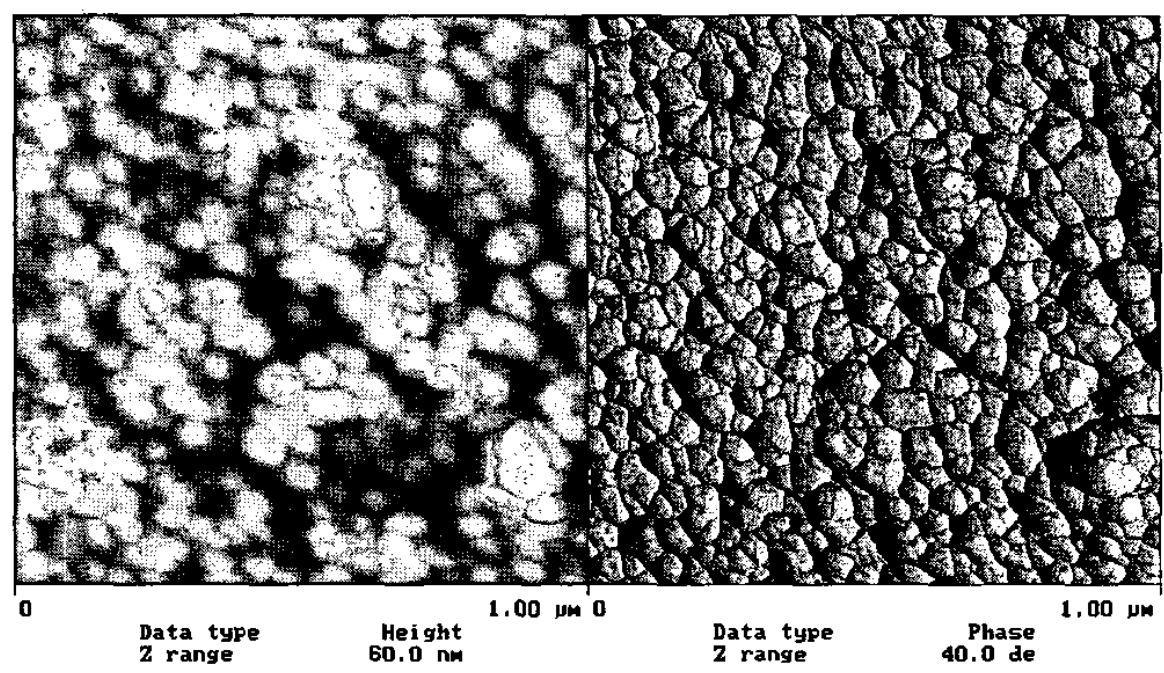

nizn.003

Fig.1. Topographic (left) and phase (right) images of the hydrothermal NiZn ferrite powders. 


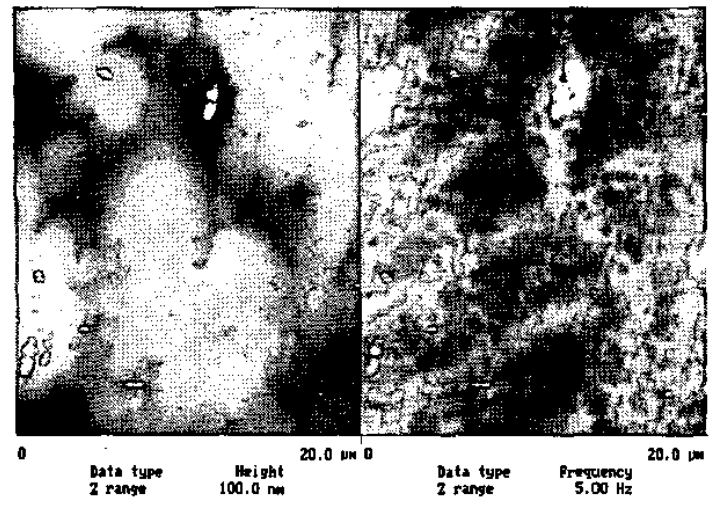

Fig.2. Topographic and magnetic images of the ferrite sintered at $1100^{\circ} \mathrm{C}$ for 4 hours.
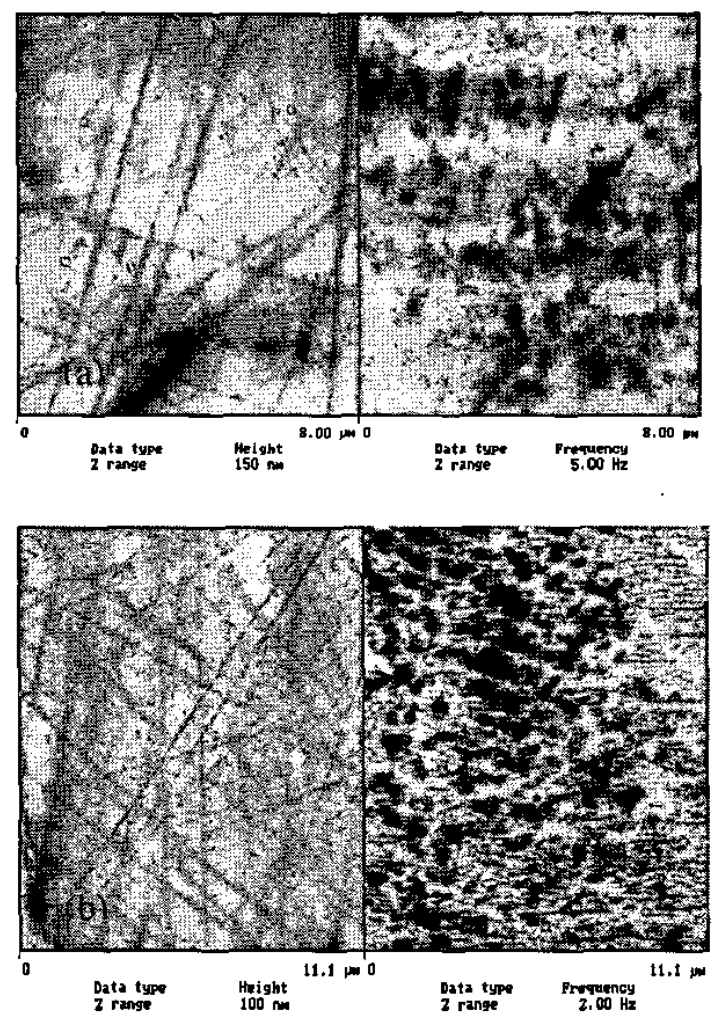

Fig.3. Topographic and magnetic images of the NiZn ferrites sintered at (a) $1300^{\circ} \mathrm{C}$ and (b) $1400^{\circ} \mathrm{C}$

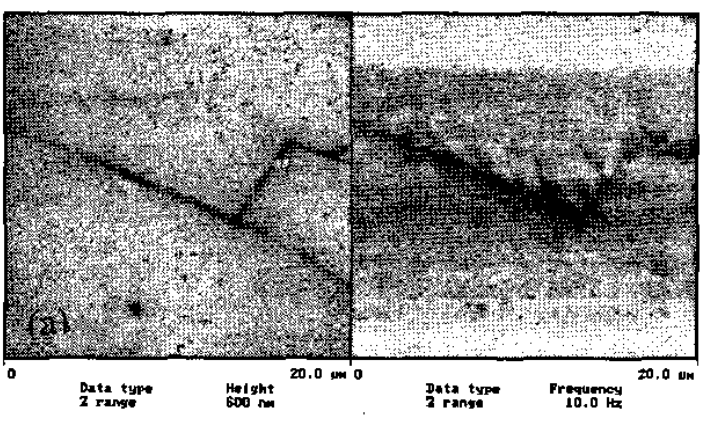

52015. 002

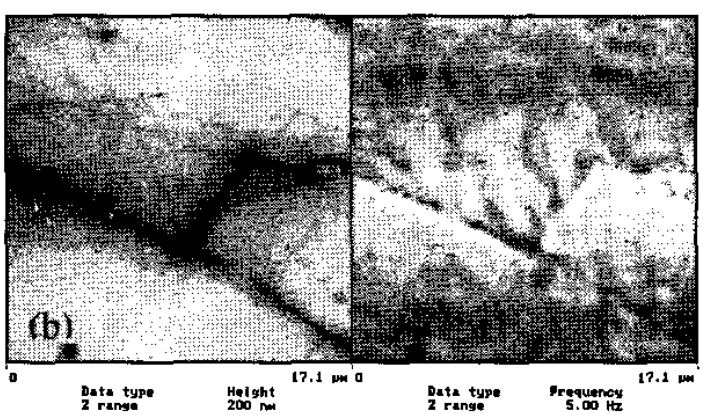

rzolm.002

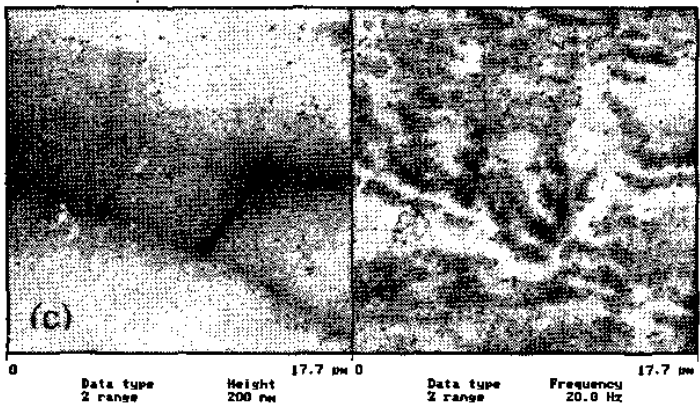

rohc. 009

Fig.4. MFM images of the hydrothermal NiZn ferrites: (a) LC, (b) LM, and (c) HC tips. 